\title{
The Effect of University Students Perceived Value on Purchasing Behavior on Online Takeaway Platforms
}

\author{
Liang Huang ${ }^{1, a, *}$ and Hui Du, ${ }^{1, b}$ \\ ${ }^{1}$ School of Economics and Management, Beijing Jiaotong University, Beijing 100044, China \\ a14125352@bjtu.edu.cn, bhdu@bjtu.edu.cn \\ ${ }^{*}$ Corresponding author
}

\begin{abstract}
Since University students are major consumers of online takeaway platforms, the factors influencing their purchasing behavior are of course a concern. Based on the literature review, the model and hypotheses about how the perceived value affects the purchasing behavior are put forward. Through questionnaires and data analysis, it is confirmed that product value, service value and redemption value put positive effect on the purchasing price, frequency, and evaluation and money cost, time cost and energy cost put negative effect on the purchasing price, frequency, and evaluation. According to the results, pertinent marketing strategies for online takeaway platforms are proposed.
\end{abstract}

Keywords: Online takeaway platforms, University students, Perceived value, Purchasing behavior, Marketing strategies.

\section{在线外卖平台大学生感知价值影响购买行为的实证研究 黄亮 ${ }^{1, a,{ }^{*}}$, 杜晖 ${ }^{1, b}$ \\ 1 北京交通大学经济管理学院信息管理系, 北京, 中国 \\ a14125352@bjtu.edu.cn, bhdu@bjtu.edu.cn \\ *通讯作者}

中文摘要. 大学生是在线外卖平台的主要 消费群体，因此，其购买行为影响因素理应 受到关注。基于文献综述, 提出了大学生感 知价值影响购买行为的模型及假设。通过问 卷调查和数据分析发现: 产品价值、服务价 值及赎回价值正向影响购买价格、购买频 率、购买评价; 货币成本、时间成本及精力 成本负向影响购买价格、购买频率、购买评 价。基于研究结果, 为在线外卖平台提供了 有针对性的营销策略建议。
关键词: 在线外卖平台; 大学生; 感知价值; 购买行为; 营销策略

\section{1. 引言}

近年来, 在线外卖平台成为电子商务的 新热点, 出现了淘点点、饿了么, 以及美团 外卖等。大学生是在线外卖平台的主要消费 群体, 因此, 其购买行为影响因素理应受到 关注。在诸多影响因素中, 顾客感知价值是 
产生积极营销的重要驱动力, 如促进重复购 买、提升企业获利能力等 ${ }^{[1]}$ 。

易法敏等将感知价值作为整体, 通过实 证研究表明: 感知价值是影响购买行为的主 要因素 ${ }^{[2]}$ 。姜参等通过实证研究发现: 感知 价值能够促进消费者的目的性和冲动性购 买, 重点强调了商店形象的影响 ${ }^{[3]}$ 。雷星晖 等对感知价值进行了细分, 通过实证研究发 现: 社会价值、功能价值、情感价值, 以及 程序价值正向影响购买意向, 但是, 研究没 有涉及感知价值的利失方面 ${ }^{[4]}$ 。综上所述, 尽管已有学者就顾客感知价值与购买行为 的关系展开了研究, 但是, 针对在线外卖平 台大学生感知价值的构成及其对购买行为 的影响研究还未见到。

本文后续内容如下：第2节在理论概述 的基础上提出了研究模型及假设; 第 3 节概 述了采集数据的问卷调查; 第 4 节介绍了数 据分析及结果; 第 5 节有针对性地为在线外 卖平台提供营销策略建议, 并指出了进一步 工作。

\section{2. 研究模型及假设}

\section{1 顾客感知价值}

对感知价值的理解分为权衡说和多因 素说。权衡说认为: 感知价值的本质是感知 利得与感知利失的权衡 ${ }^{[5]}$ 。多因素说认为: 感知价值包括获得价值、处置价值、使用价 值和赎回价值等 ${ }^{[6]}$ 。褚爱红等综合了两种学 说, 认为: 顾客购买总价值包括产品价值和 服务价值; 顾客购买总成本包括货币成本、 使用成本, 时间成本和精力成本 ${ }^{[7]}$ 。

综上所述, 作者认为: 在线外卖平台大 学生的感知价值包括感知利益和感知成本。 其中, 感知利益包括产品价值、服务价值和 赎回价值; 感知成本包括货币成本、时间成 本和精力成本。

\section{2 购买行为}

潘爆等认为: 消费者购买行为包括购买 价格和购买频率 ${ }^{[8]}$ 。余航等认为: 将购买评 价纳入购买行为有利于商家与消费者的交 流, 对销量和声誉等产生影响 ${ }^{[9]}$ 。研究表明: 正面的书籍购买评价会对书籍的销售量产
生很大帮助, 而负面的在线评论数量会显著 降低网络销售量 ${ }^{[10]}$ 。

综上所述, 作者认为: 在线外卖平台大 学生的购买行为包括购买价格、购买频率和 购买评价。

\section{3 研究模型}

根据上述分析和总结, 提出了在线外卖 平台大学生感知价值影响购买行为的研究 模型, 如图1所示。

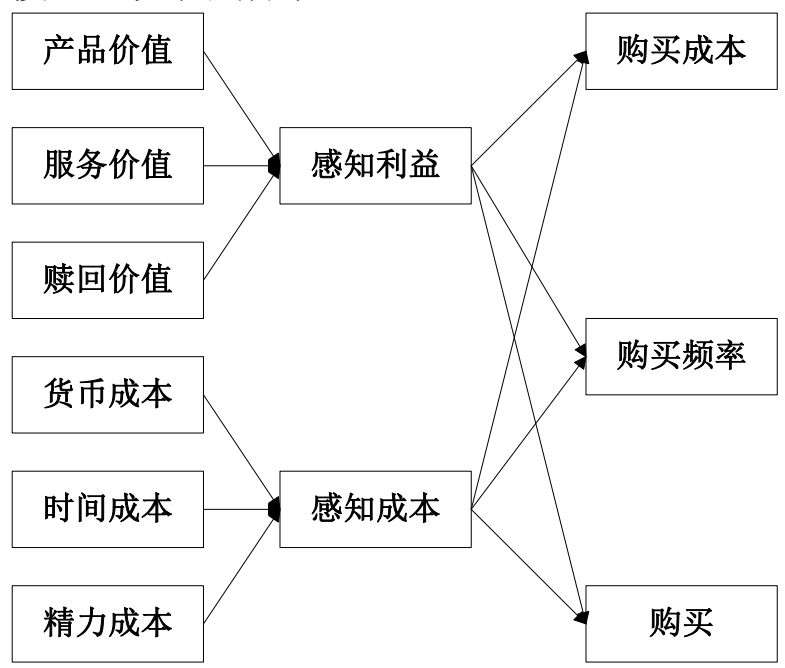

图1 在线外卖平台大学生感知价值影响购买行为的 研究模型

\section{4 研究假设}

根据研究模型, 提炼出 6 个研究假设, 如表1所示。

表1 研究假设

\begin{tabular}{|c|l|}
\hline 序号 & \multicolumn{1}{|c|}{ 假设 } \\
\hline 1 & $\begin{array}{l}\text { 大学生感知利益（包括产品、服务、赎回价值）正向影 } \\
\text { 响购买价格 }\end{array}$ \\
\hline 2 & $\begin{array}{l}\text { 大学生感知利益（包括产品、服务、赎回价值）正向影 } \\
\text { 响购买频率 }\end{array}$ \\
\hline 3 & $\begin{array}{l}\text { 大学生感知利益（包括产品、服务、赎回价值）正向影 } \\
\text { 响购买评价 }\end{array}$ \\
\hline 4 & $\begin{array}{l}\text { 大学生感知成本（包括货币、时间、精力成本）负向影 } \\
\text { 响购买价格 }\end{array}$ \\
\hline 5 & $\begin{array}{l}\text { 大学生感知成本（包括货币、时间、精力成本）负向影 } \\
\text { 响购买频率 }\end{array}$ \\
\hline 6 & $\begin{array}{l}\text { 大学生感知成本（包括货币、时间、精力成本）负向影 } \\
\text { 响购买评价 }\end{array}$ \\
\hline
\end{tabular}

\section{3. 数据采集}

问卷调查样本限定为具有在线外卖平 台购买经历的北京、上海和深圳大学生。研 究变量涉及人口统计、感知利益、感知成本 和购买行为, 除人口统计外的其他问卷调查 题目如表2所示。采用Likert5点标尺测量, 
即1表示完全不同意, 5表示完全同意。通过 网络最终回收有效问卷227份。

表2 调查问卷题目

\begin{tabular}{|c|c|}
\hline 模型变量 & $\begin{array}{c}\text { 调查题目 } \\
\end{array}$ \\
\hline \multirow[t]{3}{*}{ 产品价值 } & 1. 在线外卖平台订餐能够让我掌握更多的信息。 \\
\hline & $\begin{array}{l}\text { 2. 通过在线外卖平台能够买到色香味不错的商 } \\
\text { 品。 }\end{array}$ \\
\hline & 3. 我认为在线外卖的安全卫生有保障。 \\
\hline \multirow[t]{3}{*}{ 服务价值 } & 4. 在线外卖平台的服务有卓越的价值。 \\
\hline & 5. 在线外卖平台的页面设计很人性化。 \\
\hline & 6. 送餐人员的服务态度让我满意。 \\
\hline \multirow[t]{3}{*}{ 赎回价值 } & 7. 在线外卖可以获得较多返现。 \\
\hline & 8. 在线订餐的优惠比线下购买的多。 \\
\hline & $\begin{array}{l}\text { 9. 在线点餐第一次消费能为第二次消费带来折 } \\
\text { 扣。 }\end{array}$ \\
\hline \multirow[t]{3}{*}{ 货币成本 } & 10. 在线外卖平台的商品不是物有所值。 \\
\hline & 11. 送餐费让我觉得不合理。 \\
\hline & 12. 在线订餐是不经济的。 \\
\hline \multirow[t]{3}{*}{ 时间成本 } & $\begin{array}{l}\text { 13. 我需要花很多的时间才懂 (学会) 使用平台 } \\
\text { 订餐。 }\end{array}$ \\
\hline & 14. 我需要花很多的时间才能等到所订的餐饮。 \\
\hline & 15. 我认为商家送餐的时间与我设定的不一致。 \\
\hline \multirow[t]{3}{*}{ 精力成本 } & 16. 我觉得使用在线外卖平台很麻烦。 \\
\hline & 17. 使用网络进行订餐对我来说不太方便。 \\
\hline & 18. 取餐浪费了我很多的精力。 \\
\hline 购买价格 & 19. 我愿意付出较多的金钱来在线订餐。 \\
\hline 购买频率 & 20. 我经常通过在线外卖平台订餐。 \\
\hline 购买评价 & 21. 评价的时候我倾向于给好评。 \\
\hline
\end{tabular}

\section{4. 数据分析及结果}

\section{1 描述性统计}

通过描述性统计, 得到调查样本的人口 统计特征如表3所示。

表3 调查样本的人口统计特征

\begin{tabular}{|c|c|c|c|}
\hline 题目 & 选项 & 数量 & 百分比 \\
\hline \multirow{3}{*}{ 性别 } & 男 & 116 & $51.1 \%$ \\
\hline \multirow{4}{*}{ 年级 } & 女 & 111 & $48.9 \%$ \\
\cline { 2 - 4 } & 大一 & 45 & $19.82 \%$ \\
\cline { 2 - 4 } & 大二 & 48 & $21.15 \%$ \\
\cline { 2 - 4 } & 大三 & 50 & $22.03 \%$ \\
\cline { 2 - 4 } & 大四 & 46 & $20.26 \%$ \\
\hline 购买评价 & 研究生 & 38 & $16.74 \%$ \\
\hline \multirow{3}{*}{ 月生活费 } & 是 & 89 & $39.21 \%$ \\
\cline { 2 - 4 } (元) & 否 & 138 & $60.79 \%$ \\
\cline { 2 - 4 } & 500 以下 & 0 & 0 \\
\cline { 2 - 4 } & $500-1000$ & 73 & $32.16 \%$ \\
\cline { 2 - 4 } & $1001-1500$ & 103 & $45.37 \%$ \\
\cline { 2 - 4 } & $1501-2000$ & 45 & $19.82 \%$ \\
\hline & 2000 以上 & 6 & $2.64 \%$ \\
\hline
\end{tabular}

如表3所示: 调查样本的性别和年级分 布比较均衡; 月生活费主要集中在500-1500 元之间, 占 $77.5 \%$; $60.79 \%$ 的调查样本不做 购买评价, 未能对餐馆形成有效监督。

模型涉及变量的均值与标准差如表4所 示。
表4 变量均值与标准差

\begin{tabular}{|c|c|c|}
\hline 模型涉及变量 & 均值 & 标准差 \\
\hline 产品价值 & 3.77 & 0.855 \\
\hline 服务价值 & 3.67 & 0.776 \\
\hline 赎回价值 & 3.67 & 0.950 \\
\hline 货币成本 & 2.58 & 1.108 \\
\hline 时间成本 & 2.36 & 0.872 \\
\hline 精力成本 & 1.65 & 0.875 \\
\hline 购买价格 & 2.83 & 0.808 \\
\hline 购买频率 & 2.41 & 1.147 \\
\hline 购买评价 & 3.50 & 0.979 \\
\hline
\end{tabular}

如表4所示: 感知利益所涉及变量的均 值都大于 3 , 感知成本所涉及变量的均值都 小于 3 , 说明大学生使用在线外卖平台获得 了更多感知价值。

在感知利益所涉及变量中, “产品价值” 数据说明: 大学生对在线外卖平台的商品比 较认可。“服务价值” 数据说明: 大学生对 在线外卖平台工作人员的服务比较满意。

“赎回价值” 数据说明: 在线外卖平台相比 实体餐馆提供给大学生更多优惠。

在感知成本所涉及变量中, “货币成本” 数据说明: 大学生认为使用在线外卖平台较 为经济。“时间成本” 数据说明: 大学生认 为商家送餐较为准时。“精力成本” 数据说 明: 大学生使用在线外卖平台消耗的精力不 多。

在购买行为所涉及变量中, “购买价格” 数据说明: 大学生不愿付出更高价格。“购 买频率” 数据说明: 大学生并不经常使用在 线外卖平台。“购买评价” 数据说明: 多数 大学生能够给予好评。

\section{2 信效度分析}

使用Cronbach's Alpha指数进行信度分 析。初次检验中, “服务价值” 的Alpha值 为 0.531 。经二次检验, 发现题目 5 的校正项 总计相关性只有 0.182 , 远小于临界值 0.4 , 表明题目 5 在 “服务价值” 变量中与其他题 目相关性不高。删除题目 5, “服务价值” 的Alpha值提升为 0.641 , 感知利益、感知成 本和购买行为的Alpha值都大于 0.85 , 表明 调查数据是可靠的。

量表题目借鉴于其他学者, 已被证明具 有良好效度, 因此, 调查数据具有较好的内 容效度。感知利益、感知成本、购买行为的 KMO值均大于临界值0.6, Bartlett' s球形检 验特征值均为 0.000 , 小于显著性水平 0.05 , 因此, 调查数据具有较高的建构效度。 


\section{3 相关与回归分析}

通过计算Pearson相关系数值分析感知 利益和感知成本所涉及变量与购买行为所 涉及变量间的关系, 计算结果分别如表 5 和 表6所示。

表5 感知利益与购买行为所涉及变量Pearson值

\begin{tabular}{|c|c|c|c|c|c|c|}
\hline Pearson值 & $\begin{array}{c}\text { 产品 } \\
\text { 价值 }\end{array}$ & $\begin{array}{c}\text { 服务 } \\
\text { 价值 }\end{array}$ & $\begin{array}{c}\text { 赎回 } \\
\text { 价值 }\end{array}$ & $\begin{array}{c}\text { 购买 } \\
\text { 价格 }\end{array}$ & $\begin{array}{c}\text { 购买 } \\
\text { 频率 }\end{array}$ & $\begin{array}{c}\text { 购买 } \\
\text { 评价 }\end{array}$ \\
\hline 产品价值 & 1 & & & & & \\
\hline 服务价值 & 0.580 & 1 & & & & \\
\hline 赎回价值 & 0.619 & 0.676 & 1 & & & \\
\hline 购买价格 & 0.547 & 0.450 & 0.757 & 1 & & \\
\hline 购买频率 & 0.559 & 0.575 & 0.810 & 0.796 & 1 & \\
\hline 购买评价 & 0.878 & 0.637 & 0.656 & 0.539 & 0.599 & 1 \\
\hline
\end{tabular}

如表5所示: 感知利益所涉及变量与购 买行为所涉及变量的Pearson值均在 0.45 至 1 之间, 说明前者与后者间存在一定的正相关 关系。

表6 感知成本与购买行为所涉及变量Pearson值

\begin{tabular}{|l|c|c|c|c|c|c|}
\hline Pearson值 & $\begin{array}{c}\text { 货币 } \\
\text { 成本 }\end{array}$ & $\begin{array}{c}\text { 时间 } \\
\text { 成本 }\end{array}$ & $\begin{array}{c}\text { 精力 } \\
\text { 成本 }\end{array}$ & $\begin{array}{c}\text { 购买 } \\
\text { 价格 }\end{array}$ & $\begin{array}{c}\text { 购买 } \\
\text { 频率 }\end{array}$ & $\begin{array}{c}\text { 购买 } \\
\text { 评价 }\end{array}$ \\
\hline 货币成本 & 1 & & & & & \\
\hline 时间成本 & 0.952 & 1 & & & & \\
\hline 精力成本 & 0.827 & 0.813 & 1 & & & \\
\hline 购买价格 & $\begin{array}{c}-0.61 \\
9\end{array}$ & $\begin{array}{c}-0.64 \\
9\end{array}$ & $\begin{array}{c}-0.61 \\
0\end{array}$ & 1 & & \\
\hline 购买频率 & $\begin{array}{c}-0.70 \\
3\end{array}$ & $\begin{array}{c}-0.68 \\
9\end{array}$ & $\begin{array}{c}-0.66 \\
2\end{array}$ & 0.796 & 1 & \\
\hline 购买评价 & $\begin{array}{c}-0.77 \\
7\end{array}$ & $\begin{array}{c}-0.80 \\
4\end{array}$ & $\begin{array}{c}-0.75 \\
6\end{array}$ & 0.539 & 0.599 & 1 \\
\hline
\end{tabular}

如表6所示: 感知成本所涉及变量与购 买行为所涉及变量的Pearson值均在 -0.5 至 -1 之间, 说明前者与后者间存在一定的负相 关关系。

相关分析基本验证了研究假设的正确 性。通过回归分析, 可进一步得出感知价值 所涉及变量对购买行为所涉及变量的影响 程度, 分析结果分别如表 7 , 表8和表9所示。 表7 感知价值与购买价格的回归分析

\begin{tabular}{|c|c|c|c|c|c|c|}
\hline 变量 & \multicolumn{7}{|c|}{ 购买价格 } \\
\cline { 2 - 7 } & $\begin{array}{c}\text { 常数 } \\
\text { 项 }\end{array}$ & 系数 & $\mathrm{F}$ & Sig. & $\mathrm{R}$ 方 & $\begin{array}{c}\text { 调整 } \mathrm{R} \\
\text { 方 }\end{array}$ \\
\hline $\begin{array}{l}\text { 产品 } \\
\text { 价值 }\end{array}$ & 0.306 & 0.547 & 96.026 & 0.000 & 0.299 & 0.296 \\
\hline $\begin{array}{l}\text { 服务 } \\
\text { 价值 }\end{array}$ & 0.708 & 0.450 & 57.002 & 0.000 & 0.202 & 0.199 \\
\hline $\begin{array}{l}\text { 赎回 } \\
\text { 价值 }\end{array}$ & -1.12 & 0.757 & $\begin{array}{c}302.11 \\
9\end{array}$ & 0.000 & 0.573 & 0.571 \\
\hline $\begin{array}{l}\text { 货币 } \\
\text { 成本 }\end{array}$ & 4.154 & $\begin{array}{c}-0.61 \\
9\end{array}$ & $\begin{array}{c}139.75 \\
6\end{array}$ & 0.000 & 0.383 & 0.380 \\
\hline $\begin{array}{l}\text { 时间 } \\
\text { 成本 }\end{array}$ & 4.503 & $\begin{array}{c}-0.64 \\
9\end{array}$ & $\begin{array}{c}163.72 \\
7\end{array}$ & 0.000 & 0.421 & 0.419 \\
\hline $\begin{array}{l}\text { 精力 } \\
\text { 成本 }\end{array}$ & 3.843 & $\begin{array}{c}-0.61 \\
0\end{array}$ & $\begin{array}{c}133.37 \\
2\end{array}$ & 0.000 & 0.372 & 0.369 \\
\hline
\end{tabular}

表7说明回归效果显著。“调整R方” 数据表明: 产品价值、服务价值、赎回价值、 货币成本、时间成本、精力成本分别解释了 购买价格变异的 $29.6 \% 、 19.9 \% 、 57.1 \%$ 、 $38.0 \% 、 41.9 \% 、 36.9 \%$ 。

表8 感知价值与购买频率的回归分析

\begin{tabular}{|c|c|c|c|c|c|c|}
\hline \multirow{2}{*}{ 变量 } & \multicolumn{7}{|c|}{ 购买频率 } \\
\cline { 2 - 7 } & $\begin{array}{c}\text { 常数 } \\
\text { 项 }\end{array}$ & 系数 & $\mathrm{F}$ & $\mathrm{Sig}$. & $\mathrm{R}$ 方 & $\begin{array}{c}\text { 调整 } \\
\text { R方 }\end{array}$ \\
\hline $\begin{array}{c}\text { 产品 } \\
\text { 价值 }\end{array}$ & -1.337 & 0.559 & 102.334 & 0.000 & 0.313 & 0.310 \\
\hline $\begin{array}{c}\text { 服务 } \\
\text { 价值 }\end{array}$ & -1.519 & 0.575 & 111.004 & 0.000 & 0.330 & 0.327 \\
\hline $\begin{array}{c}\text { 赎回 } \\
\text { 价值 }\end{array}$ & -2.177 & 0.810 & 428.721 & 0.000 & 0.656 & 0.654 \\
\hline $\begin{array}{c}\text { 货币 } \\
\text { 成本 }\end{array}$ & 4.620 & -0.703 & 219.998 & 0.000 & 0.494 & 0.492 \\
\hline $\begin{array}{l}\text { 时间 } \\
\text { 成本 }\end{array}$ & 5.012 & -0.689 & 202.921 & 0.000 & 0.474 & 0.472 \\
\hline $\begin{array}{l}\text { 精力 } \\
\text { 成本 }\end{array}$ & 4.028 & -0.662 & 175.890 & 0.000 & 0.439 & 0.436 \\
\hline
\end{tabular}

表8说明回归效果显著。“调整R方” 数据表明: 产品价值、服务价值、赎回价值、 货币成本、时间成本、精力成本分别解释了 购买频率变异的 $31.0 \% 、 32.7 \% 、 65.4 \%$ 、 $49.2 \% 、 47.2 \% 、 43.6 \%$ 。

表9 感知价值与购买评价的回归分析

\begin{tabular}{|c|c|c|c|c|c|c|}
\hline \multirow{2}{*}{ 变量 } & \multicolumn{7}{|c|}{ 购买评价 } \\
\cline { 2 - 7 } & $\begin{array}{c}\text { 常数 } \\
\text { 项 }\end{array}$ & 系数 & $\mathrm{F}$ & Sig. & $\mathrm{R}$ 方 & $\begin{array}{c}\text { 调整 } \\
\text { R方 }\end{array}$ \\
\hline $\begin{array}{c}\text { 产品 } \\
\text { 价值 }\end{array}$ & -0.842 & 0.878 & 753.290 & 0.000 & 0.770 & 0.769 \\
\hline $\begin{array}{c}\text { 服务 } \\
\text { 价值 }\end{array}$ & 0.298 & 0.637 & 153.545 & 0.000 & 0.406 & 0.403 \\
\hline $\begin{array}{c}\text { 赎回 } \\
\text { 价值 }\end{array}$ & 0.774 & 0.656 & 169.876 & 0.000 & 0.430 & 0.428 \\
\hline $\begin{array}{c}\text { 货币 } \\
\text { 成本 }\end{array}$ & 5.423 & -0.777 & 343.702 & 0.000 & 0.604 & 0.603 \\
\hline $\begin{array}{l}\text { 时间 } \\
\text { 成本 }\end{array}$ & 5.873 & -0.804 & 410.469 & 0.000 & 0.646 & 0.644 \\
\hline $\begin{array}{l}\text { 精力 } \\
\text { 成本 }\end{array}$ & 4.976 & -0.756 & 301.053 & 0.000 & 0.572 & 0.570 \\
\hline
\end{tabular}

如表9，整个回归效果显著。“调整R 方” 数据说明产品价值、服务价值、赎回价 值、货币成本、时间成本、精力成本分别解 释了购买评价变异的 $76.9 \% 、 40.3 \% 、 42.8 \%$ 、 $60.3 \% 、 64.4 \% 、 57.0 \%$ 。

上述相关与回归分析结果表明：图1提 出的模型和表 1 提出的假设都是成立的。

\section{5. 结束语}

基于上述分析结果, 结合市场营销的4P 及 $4 \mathrm{C}$ 理论, 为现有在线外卖平台提出了针 对性的营销策略建议: 在 “产品” 和 “顾客” 方面丰富产品种类，提供贴心细致服务，增 
加产品与服务价值; 在 “价格” 和 “成本” 方面开发优惠产品, 准时送餐, 降低货币与 时间成本; 在 “渠道” 和 “便利” 方面全方 位宣传, 提供多种下单方式, 降低精力成本; 在 “促销” 和 “沟通” 方面举办多种优惠活 动, 提高赎回价值, 建立有效互动机制, 促 进购买评价。

扩大研究样本数量, 增强代表性; 将营 销策略建议应用到具体在线外卖平台, 验证 其有效性是下一步研究的重点。

\section{References}

[1] CHEN Na and HOU Guang-hui, Library User's Perceived Value and Participation Willingness in the Subject Service: the Moderating Role of Communication Service, Science and Technology Management Research, vol.33, pp. 230-235, 2013.

[2] YI Fa-min and FAN Gao-feng, An Empirical Study on the Effects of Consumer Purchase Intention on Online Shopping Behavior, Humanities \& Social Sciences Journal of Hainan University, vol.28, pp. 66-73, 2010.

[3] JIANG Shen and ZHAO Hong-xia, Online-store Image, Customer Perceived and Purchasing Behavior, Research on Financial and Economic Issues, vol.10, pp. 116-122, 2013.

[4] LEI Xing-hui and ZHANG Wei, Influence of Customer Perceived Value on Purchase Behavior and Enterprise
Future Sales on Electronic Commerce Platform, Shanghai Management Science, vol. 04, pp. 27-33, 2012.

[5] WANG Nai-jing and DU Tao, Customer Value and Structuring of Customer Value Chain, Value Engineering, vol. 23, pp. 28-31, 2004.

[6] A. PARASURAMAN and DHRUV GREWAL, Serving customers and Consumers Effectively in the Twenty-first Century: a Conceptual Framework and Overview, Journal of the Academy of Marketing Science, vol. 28, pp. 9-16, 2000.

[7] ZHU Ai-hong, The Analyze of Customer Value Management, On Economic Problems, vol. 08, pp. 54-55, 2006.

[8] PAN Yu, GAO Li and WANG Fang-hua, Influences of Lifestyle and Customer Perceived Values on Chinese Consumer Purchasing Behaviors, Journal of Systems \& Management, vol. 06, pp. 601-607, 2009.

[9] YU Hang and WANG Zhan-ping, R esearch on the Effects of Online Word-of-Mouth Communication, Journal of Intelligence, vol. 32, pp.100-106, 2013.

[10]CHECALIER J.D. MAYZLIN, The Effect of Word of Mouth on Sales: Online Book Reviews, Journal of Marketing Research, vol. 08, pp. 345-354, 2006. 